\title{
Le carnet pour lui-même, dir. F. DUMONT
}

\section{Gabriella Bosco}

\section{CpenEdition \\ Journals}

\section{Edizione digitale}

URL: https://journals.openedition.org/studifrancesi/23377

DOI: 10.4000/studifrancesi.23377

ISSN: 2427-5856

\section{Editore}

Rosenberg \& Sellier

\section{Edizione cartacea}

Data di pubblicazione: 1 avril 2020

Paginazione: 234-235

ISSN: 0039-2944

\section{Notizia bibliografica digitale}

Gabriella Bosco, «Le carnet pour lui-même, dir. F. Dumont», Studi Francesi [Online], 190 (LXIV | I) | 2020, online dal 01 mai 2020, consultato il 03 août 2021. URL: http://journals.openedition.org/studifrancesi/ 23377 ; DOI: https://doi.org/10.4000/studifrancesi.23377

Questo documento è stato generato automaticamente il 3 août 2021.

\section{(c) (†)}

Studi Francesi è distribuita con Licenza Creative Commons Attribuzione - Non commerciale - Non opere derivate 4.0 Internazionale. 


\title{
Le carnet pour lui-même, dir. F. DUMONT
}

\author{
Gabriella Bosco
}

\section{NOTIZIA}

Le carnet pour lui-même, dir. F. DUMONT, Université Laval, 2019, “Études littéraires” 48, 1-2, $202 \mathrm{pp}$.

1 Molto a lungo i carnets degli scrittori sono stati considerati testi utili allo studio della loro opera, in qualche modo preparatori, retro-bottega interessanti da leggere per le informazioni che potevano contenere rispetto a qualcosa di futuro o di cui avevano accompagnato la genesi. Oggi li si considera invece, a buon diritto, testi à part entière che, pur preservando gli aspetti legati alla loro dimensione in buona parte di laboratorio, hanno acquisito il diritto all'autonomia, all'esistenza in quanto tali.

Questo è il senso del titolo che il curatore del doppio fascicolo di «Études littéraires» dedicato a questo genere di scrittura, François DumonT, gli ha voluto dare: il carnet considerato per se stesso, a prescindere da ogni altra sua funzione. Nelle pagine di presentazione (pp. 7-12), Dumont opera anche una distinzione rispetto a forme di scrittura limitrofe a quella del carnet, in particolare il cahier e il journal, più studiati da un punto di vista critico, per la loro comune appartenenza al ventaglio autobiografico.

Due gli obiettivi enunciati in apertura: quello di risalire alle origini dell'"esprit du carnet" a partire dall'Antichità; e quello di esplorare la diversità delle sue forme contemporanee. Entrambi perseguiti attraverso una molteplicità di approcci: dalla contestualizzazione storica allo studio della poetica, del genere o della genesi.

Il primo contributo, di Andrei MINZETANU (Le carnet du littré: objet matériel, objet mental, pp. 13-18), riflette sui numerosi enjeux teorici della forma del carnet, dal punto di visto della sua concreta realizzazione come da quello dell'atteggiamento mentale che presiede alla sua scrittura. Fanno seguito una serie di interventi consacrati ai 
progenitori del carnet. Inizia Pascale FLEURY (Les discours pour soi et sur soi dans l'Antiquité: les pratiques de l'intime dans les "Pensées" et les lettres de Marc Aurèle et chez quelques prédécesseurs, pp. 19-32), che studia testi dell'imperatore romano spesso assimilati agli hypomnemata, note di generali e magistrati caratterizzate dalla loro natura transitoria e documentaria, destinate a essere trasformate in testi più rifiniti, e però di per sé stessi, secondo l'A., definitori dell'io di chi scrive. In questo senso, afferma Pascale Fleury, Marco Aurelio anticipa le pratiche contemporanee di coloro che fanno del carnet un uso letterario.

5 Evelyne LESIGNE-AUDOLY si occupa invece delle Notes de chevet lungamente attribuite a Sei Shônagon, dama di corte al servizio dell'imperatrice Teishi, una delle due imperatrici dell'epoca, entrambe mogli dell'imperatore Ichijô - in giapponese Makura no sôshi, letteralmente "libro-cuscino" - testo dell'anno 1000 circa, sequenza di trecento corti brani diversi per forma e contenuto, che ha avuto larga diffusione in Occidente ispirando scrittori e artisti, francesi tra gli altri (da Roland Barthes a Georges Perec, François Bon, Charles Dantzig, Chris Marker, Pascal Quignard, Jean-Claude Carrière) che vi hanno scoperto la possibilità di una scrittura discontinua e frammentaria. Dagli anni Ottanta del Novecento, gli storici della letteratura giapponesi relativizzano la natura intimista delle Notes de chevet e insistono maggiormente sul carattere ufficiale e quasi pubblico dell'attività letteraria che sta dietro al testo, di cui Sei Shônagon avrebbe in realtà redatto una sorta di registro utilizzando risme di carta ricevute in dono (Des liasses de papier dont on fit un "oreiller": le registre de l'écriture de Sei Shônagon, pp. 33-48). L'aspetto concreto, materiale, del carnet messo in luce nel contributo di Evelyne Lesigne-Audoly, torna nel successivo, di Laurent GERBIER e Irène LANGLET, che - in mancanza di un manoscritto degli Essais, mai ritrovato - studiano le versioni annotate del testo, gli "allongeails", dispositivo di tipo carnettiste, scrivono gli A., nato dopo invece che prima del testo (Montaigne carnettiste, pp. 49-65, contributo arricchito dall'esemplificazione fotografica del griffonnage di Montaigne). Ai carnets di Joseph Joubert, redatti in piena tormenta rivoluzionaria, intorno al 1790, dedica il suo intervento Étienne BEAULIEU, note accompagnate spesso da piccoli disegni e schizzi, scrittura sulla cui novità lo stesso Joubert si interroga (visto da Maurice Blanchot come all'origine del livre à venir), "idee cave" nate per essere scavate fino a consunzione, in palese controtendenza rispetto alla coeva retorica di epoca rivoluzionaria e imperiale (Des tessons éparpillés, pp. 67-74).

6 Con Isabelle DAUNAIS e il suo articolo sui carnet di Julien Gracq si passa invece alla contemporaneità, cui è dedicata tutta la seconda parte del fascicolo. La scrittura di Gracq è duplice, spiega l'A., distinguendo un Gracq "créateur", quello dei romanzi, e un Gracq "lecteur" (di libri come di paesaggi), quello dei carnets. Ma riformulando poi la distinzione, per parlare piuttosto di due tipi di creazione, e definire quella frammentaria del carnet come spazio "sospeso e staccato", distinto dallo spazio romanzesco (Les carnets de Julien Gracq: "la promenade entre toutes préférée", pp. 75-85). Mentre Sophie HÉBERT studia la specificità dei carnets di Henri Thomas, da lui usati per raccogliervi le note rapide, i pensieri allo stadio nascente, ma anche come luogo in cui autobiografia, poesia e saggio si trovano riconciliati e in qualche modo amalgamati (Les "écritures confuses" d'Henri Thomas: perspectives poétique, générique, génétique, pp. 87-102). L'intervento successivo, di Christophe MEURÉE, analizza la funzione del carnet (o meglio, nel suo caso, piuttosto del cahier) per Henry Bauchau, che se ne serve per costruirvi la propria postura di scrittore anche attraverso la pratica del collage di immagini, 
riproduzioni di opere d'arte, fotografie, utili a orientare oltre che se stesso anche lettori e esegeti (Des fenêtres sur l'infini? Usages du cahier dans la construction de la posture chez Henry Bauchau, pp. 103-116). Per Aimé Césaire, di cui si occupa poi Olga HEL-BONGO, carnet e cahier si fondono in una scrittura unica che mescola l'annotazione rapida alla riflessione sul processo creativo, la pratica della stesura preparatoria a quella del frammento svelatore, in un'ottica di rispecchiamento della natura intrinsecamente polifonica della sua opera (Poétique du carnet dans "Cahier d'un retour au pays natal" d'Aimé Césaire, pp. 119-135).

7 I tre contributi che seguono riguardano autori della generazione successiva, nati negli anni Quaranta: Peter Handke, André Major e Jean-Pierre Issenhuth. Robert DioN battezza "carnet-journal" Le poids du monde di Handke, primo libro di una serie che ne conta per il momento cinque, sorta di scrittura reportage e insieme trascrizione «d'une conscience au moment même où elle prend vie dans la langue», abolizione della frontiera tra esterno e interno (Créer une forme de l'informe: "Le poids du monde" de Peter Handke, pp. 137-151). Secondo Michel BIRON invece André Major, canadese del Québec, pur inserendosi da un lato in una tradizione dell'uso del carnet meno sperimentale, quella che porta da Jules Renard a Cesare Pavese, ne fa d'altro lato anche il luogo d'esercizio di una personale forma di critica letteraria e contemporaneamente quello di un andirivieni continuo tra vita e scrittura, all'insegna dell'idea di diserzione, del mondo per scrivere e della scrittura per vivere (Le fil du carnet chez André Major, pp. 153-163). Thomas Mainguy studia infine l'uso del carnet da parte di Jean-Pierre Issenhuth il quale, allo scopo di "abitare altrimenti il mondo" (l'espressione è di Biron), si serve della scrittura carnettistica per abbandonare la poesia, linguaggio che considera troppo artificiale per permettere un contatto diretto con il reale, la natura e gli animali, tenendo a mente la nozione di radicamento definita da Simone Weil (L'enracinement de Jean-Pierre Issenhuth, pp. 165-176). In solo apparente contraddizione con il ritorno alla terra di Issenhuth, l'ultimo intervento è dedicato al carnet digitale: solo apparente perché anche nell'universo virtuale il dato costitutivo della scrittura dell'istante rimane la sua concretezza che, nonostante cambi il supporto, determina un rapporto di continuità e complementarietà, afferma René Audet, tra il carnet tradizionale e quello informatico (Penser les carnets numériques d'écrivain: écritures médiatisées et réinvestissement de l'idée de publication, pp. 177-190).

Un numero di "Études littéraires" particolarmente ricco, questo, costruito intorno alla visione della scrittura come di un processo in fieri di cui il carnet si fa portavoce. Arricchiscono la varietà dei contributi le pagine di riferimenti bibliografici che ognuno di essi comporta. 